Submitted to the Annals of Statistics

\title{
ON SEMIDEFINITE RELAXATIONS FOR THE BLOCK MODEL
}

\author{
By Arash A. Amini ${ }^{\ddagger}$ And Elizaveta LevinA ${ }^{\S}$ \\ $\ddagger U C L A$ and ${ }^{\S}$ University of Michigan
}

This supplement contains proofs of the remaining results, a detailed description of the implementation of an ADMM solver for SDP 1, and additional details on simulations.

\section{Proofs of Section 4.2.1.}

Proof of Lemma 4.1. Let $\boldsymbol{S}_{0}:=\operatorname{supp}\left(X_{0}\right)$. We proceed in two steps, first setting elements on $\boldsymbol{S}_{0}$ to one, and then setting elements on $\boldsymbol{S}_{0}^{c}$ to zero. More precisely, let $\widetilde{A}_{1}=\widetilde{A}$ on $\boldsymbol{S}_{0}$ (meaning that $\left[\widetilde{A}_{1}\right]_{i j}=\widetilde{A}_{i j}$ for $(i, j) \in \boldsymbol{S}_{0}$ ) and $\widetilde{A}_{1}=A$ on $\boldsymbol{S}_{0}^{c}$. Let $X$ be any feasible solution other than $X_{0}$, so that $0 \leq X \leq 1$. We will use the notation $\langle A, X\rangle_{\boldsymbol{S}_{0}}:=\sum_{(i, j) \in \boldsymbol{S}_{0}} A_{i j} X_{i j}$. By (unique) optimality of $X_{0}$ for $A$, we have $\langle A, X\rangle\left\langle\left\langle A, X_{0}\right\rangle\right.$. Then,

$$
\left\langle\widetilde{A}_{1}, X-X_{0}\right\rangle_{\boldsymbol{S}_{0}^{c}}=\left\langle A, X-X_{0}\right\rangle_{\boldsymbol{S}_{0}^{c}}\left\langle\left\langle A, X_{0}-X\right\rangle_{\boldsymbol{S}_{0}} \leq\left\langle\widetilde{A}_{1}, X_{0}-X\right\rangle_{\boldsymbol{S}_{0}}\right.
$$

where the first equality is by assumption and the last inequality follows from $A \leq \widetilde{A}_{1}$ on $\boldsymbol{S}_{0}$, and that $X_{0}-X \geq 0$ on $\boldsymbol{S}_{0}$. (Note that $X_{0}=\boldsymbol{E}_{m}$ on $\boldsymbol{S}_{0}$ and $X \leq 1$ everywhere.) Hence, the conclusion of the lemma follows for $\widetilde{A}_{1}$. Now, we can write

$$
\langle\widetilde{A}, X\rangle \leq\left\langle\widetilde{A}_{1}, X\right\rangle<\left\langle\widetilde{A}_{1}, X_{0}\right\rangle=\left\langle\widetilde{A}, X_{0}\right\rangle .
$$

The first inequality is by nonnegativity of $X$ and $\widetilde{A} \leq \widetilde{A}_{1}$ everywhere. The second inequality is by (unique) optimality of $X_{0}$ for $\widetilde{A}_{1}$. The last equality is by $\widetilde{A}=\widetilde{A}_{1}$ on $\boldsymbol{S}_{0}$.

Proof of Corollary 4.2. We construct a coupling between $A$ and $\widetilde{A}$. Recall that $S_{k}$ denotes the indices of nodes in community $k$. Draw $A \sim$ $\operatorname{BM}_{m}^{\text {bal }}(\Psi)$, and draw

$$
\begin{array}{ll}
R_{i j} \sim \operatorname{Bern}\left(\frac{\widetilde{p}_{k}-p_{k}}{1-p_{k}}\right), \quad(i, j) \in S_{k}, \forall k \\
R_{i j} \sim \operatorname{Bern}\left(\widetilde{q}_{k \ell} / q_{k \ell}\right), \quad(i, j) \in S_{k} \times S_{\ell}, \forall k<\ell
\end{array}
$$


independently from $A$. Extend $R$ symmetrically, by setting $R_{S_{k} S_{\ell}}=R_{S_{k} S_{\ell}}^{T}$ for $k>\ell$. Let

$$
\begin{array}{ll}
\widetilde{A}_{i j}:=1-\left(1-A_{i j}\right)\left(1-R_{i j}\right), & (i, j) \in S_{k}, \forall k \\
\widetilde{A}_{i j}:=A_{i j} R_{i j}, & (i, j) \in S_{k} \times S_{\ell}, \forall k<\ell
\end{array}
$$

and extend symmetrically. It is easy to verify that $\widetilde{A}$ has distribution $\mathrm{BM}_{m}^{\mathrm{bal}}(\widetilde{\Psi})$. Moreover, by construction $\widetilde{A} \geq A$ on $\operatorname{supp}\left(X_{0}\right)$ and $\widetilde{A} \leq A$ on $\operatorname{supp}\left(X_{0}\right)^{c}$. The result now follows from Lemma 4.1.

10. Proof of Lemma 4.2. To prove the lemma, we need the following intermediate result.

Lemma 10.1. Let $X \in \mathbb{S}^{n}$ with $\operatorname{range}(X) \subset \operatorname{span}\left\{\mathbf{1}_{S_{k}}\right\}$. Then $X=$ $B \otimes \boldsymbol{E}_{m}$ for some $B \in \mathbb{S}^{K}$, that is, $X$ is block-constant.

Proof. Note that $\mathbf{1}_{S_{k}}=e_{k} \otimes \mathbf{1}_{m}$ where $e_{k}=e_{k}^{K}$ is the $k$-th basis vector of $\mathbb{R}^{K}$. An eigenvector $v_{j}$ of $X$ will be of the form $v_{j}=\sum_{k} \alpha_{k}^{j} \mathbf{1}_{S_{k}}=\left(\sum \alpha_{k}^{j} e_{k}\right) \otimes$ $\mathbf{1}_{m}=u_{j} \otimes \mathbf{1}_{m}$ for some $u_{j} \in \mathbb{R}^{K}$. Then,

$$
\begin{aligned}
X=\sum_{j} \beta_{j} v_{j} v_{j}^{T} & =\sum_{j} \beta_{j}\left(u_{j} \otimes \mathbf{1}_{m}\right)\left(u_{j} \otimes \mathbf{1}_{m}\right)^{T} \\
& =\sum_{j} \beta_{j}\left(u_{j} u_{j}^{T}\right) \otimes\left(\mathbf{1}_{m} \mathbf{1}_{m}^{T}\right)=\left(\sum_{j} \beta_{j} u_{j} u_{j}^{T}\right) \otimes \boldsymbol{E}_{m} .
\end{aligned}
$$

Proof of Lemma 4.2.. Conditions (A1) and (A2) together satisfy (CSa) and $(\mathrm{CSb})$ for $X_{0}$ and $(\mu, \nu, \Gamma)$, in addition to dual feasibility. Hence, $X_{0}$ is an optimal solution of the primal problem. To show uniqueness, let $X$ be any optimal primal solution. Then $X$ and the specific triple $(\mu, \nu, \Gamma)$ assumed in the statement of the lemma should together satisfy optimality conditions. (CSb) for $X$ (and the triple) implies

$$
\operatorname{range}(X) \subset \operatorname{ker}(\Lambda(\mu, \nu, \Gamma))=\operatorname{span}\left\{\mathbf{1}_{S_{k}}\right\}
$$

by (A1), which then implies $X=B \otimes \boldsymbol{E}_{m}$ for some $B=\left(b_{k \ell}\right) \in \mathbb{S}^{K}$ by Lemma 10.1. Note that this means $X_{S_{k} S_{\ell}}=b_{k \ell} \boldsymbol{E}_{m}$. Now, (CSa) for $X$ implies

$$
0=X_{S_{k} S_{\ell}} \circ \Gamma_{S_{k} S_{\ell}}=b_{k \ell} \Gamma_{S_{k} S_{\ell}}, \quad \text { for } k \neq \ell
$$

using $\boldsymbol{E}_{m} \circ D=D$, for any $D$. But since $\Gamma_{S_{k} S_{\ell}}$ is not identically zero by (A3), we should have $b_{k \ell}=0$, for $k \neq \ell$. One the other hand, primal feasibility of $X$, in particular, $X_{i i}=1$ implies $b_{k k}=1$. That is, $B=I_{K}$, hence $X=I_{K} \otimes \boldsymbol{E}_{m}=X_{0}$. 
10.1. Proof of Theorem 4.1: primal-dual witness for SDP-2'. For SDP$2^{\prime}$, the linear condition $\mathcal{L}_{2}(X)=b_{2}$ is just the scalar equation $\left\langle\boldsymbol{E}_{n}, X\right\rangle=$ $n^{2} / K=n m$. The dual variable $\mu$ is a scalar in this case, and we have $b_{2}=m n$ and $\mathcal{L}_{2}^{*}(\mu)=\mu \boldsymbol{E}_{n}$. Hence, we have (cf. (4.10))

$$
\Lambda=\Lambda(\mu, \nu, \Gamma)=\mu \boldsymbol{E}_{n}+\operatorname{diag}^{*}(\nu)-A-\Gamma .
$$

Let $d\left(S_{k}\right)=A \mathbf{1}_{S_{k}}$ be the vector of node degrees relative to subgraph $S_{k}$. We denote its $i$ th element by $d_{i}\left(S_{k}\right)=\sum_{j \in S_{k}} A_{i j}$. Let $P_{\mathbf{1}_{m}^{\perp}}:=I_{m}-\frac{1}{m} \boldsymbol{E}_{m}$ be projection onto $\operatorname{span}\left\{\mathbf{1}_{m}\right\}^{\perp}$. The following summarizes our primal-dual construction, modulo the choice of $\mu$ :

$$
\begin{aligned}
\nu_{i} & :=d_{i}\left(S_{k}\right)-\mu m, \quad \text { for } i \in S_{k}, \\
\Gamma_{S_{k}} & :=0, \quad \forall k \\
\Gamma_{S_{k} S_{\ell}} & :=\mu \boldsymbol{E}_{m}+P_{\mathbf{1}_{m}^{\frac{1}{m}}} A_{S_{k} S_{\ell}} P_{\mathbf{1}_{m}^{\perp}}-A_{S_{k} S_{\ell}},
\end{aligned}
$$

for all $k \neq \ell$. Note that $\Gamma$ is symmetric. Let

$$
\Delta:=A-\mathbb{E}[A], \quad d_{\mathrm{av}}\left(S_{k}, S_{\ell}\right):=\frac{1}{m} \sum_{i \in S_{k}} d_{i}\left(S_{\ell}\right)=\frac{1}{m} \sum_{j \in S_{\ell}} d_{j}\left(S_{k}\right) .
$$

The following lemma, proved in Section 11, verifies the validity of this construction.

Lemma 10.2. Let $(\mu, \nu, \Gamma)$ be as in (10.2) and (10.3). Then, $\Gamma$ verifies (A2) and (4.11) holds for all $\mu$. In addition,

(a) $\Gamma$ is dual feasible, i.e. $\Gamma \geq 0$, if for all $i \in S_{k}, j \in S_{\ell}, k \neq \ell$,

$$
\mu m \geq d_{i}\left(S_{\ell}\right)+d_{j}\left(S_{k}\right)-d_{a v}\left(S_{k}, S_{\ell}\right),
$$

and satisfies (A3) if at least one inequality is strict for each pair $k \neq \ell$.

(b) $\Gamma$ verifies $(4.12)$ if

$$
(\rho-\mu) m>\|\Delta\|, \quad \text { where } \rho:=\min _{k} \min _{i \in S_{k}} d_{i}\left(S_{k}\right) / m .
$$

We note that choosing $\mu m$ to be the maximum of the RHS of (10.5), i.e.,

$$
\max _{k, \ell} \max _{i \in S_{k}, j \in S_{\ell}}\left[d_{i}\left(S_{\ell}\right)+d_{j}\left(S_{k}\right)-d_{\mathrm{av}}\left(S_{k}, S_{\ell}\right)\right]
$$

together with (10.6) gives a deterministic condition for the success of SDP$2^{\prime}$. In Section 12, we give a probabilistic version of this condition which completes the proof of Theorem 4.1. 
11. Proof of Lemma 10.2. We start by seeing how far the KKT conditions determine the dual variables and how much freedom in choosing them is left. In accordance with condition (A2), we set $\Gamma_{S_{k}}:=0$. Then, (4.11) holds if and only if $\Lambda_{S_{k}} \mathbf{1}_{m}=0$ and $\Lambda_{S_{k}^{c} S_{k}} \mathbf{1}_{m}=0$, or equivalently,

$$
\Lambda_{S_{k}} \mathbf{1}_{m}=\left(\mu \boldsymbol{E}_{m}+\operatorname{diag}^{*}\left(\nu_{S_{k}}\right)-A_{S_{k}}\right) \mathbf{1}_{m}=\mu m \mathbf{1}_{m}+\nu_{S_{k}}-A_{S_{k}} \mathbf{1}_{m} \quad=0
$$

$$
\Lambda_{S_{k}^{c} S_{k}} \mathbf{1}_{m}=\left[\mu \boldsymbol{E}_{n-m, m}-(A+\Gamma)_{S_{k}^{c} S_{k}}\right] \mathbf{1}_{m}=\mu m \mathbf{1}_{n-m}-(A+\Gamma)_{S_{k}^{c} S_{k}} \mathbf{1}_{m}=0
$$

Let $d\left(S_{k}\right)=A \mathbf{1}_{S_{k}}$ be the vector of node degrees relative to community/subgraph $S_{k}$. We denote its $i$ th element as $d_{i}\left(S_{k}\right)=\sum_{j \in S_{k}} A_{i j}$. Note also that $A_{S_{k}} \mathbf{1}_{m}=$ $\left[d\left(S_{k}\right)\right]_{S_{k}}$. Then, setting $\nu_{i}:=d_{i}\left(S_{k}\right)-\mu m$ for $i \in S_{k}$ verifies (11.1). To verify (11.2), we need to have

$$
(A+\Gamma)_{S_{k} S_{\ell}} \mathbf{1}_{m}=\mu m \mathbf{1}_{m}, \quad \text { for all } \ell \neq k .
$$

Note that the same holds for $(A+\Gamma)_{S_{\ell} S_{k}}$. That is, every row and column of

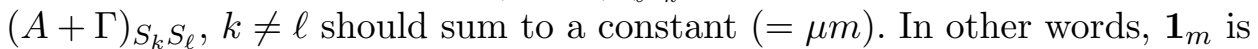
a right and left eigenvector of $(A+\Gamma)_{S_{k} S_{\ell}}$ associated with eigenvalue $\mu \mathrm{m}$. By spectral theorem (i.e., SVD), we should have

$$
(A+\Gamma)_{S_{k} S_{\ell}}=\mu \boldsymbol{E}_{m}+B_{S_{k} S_{\ell}}
$$

where $B_{S_{k} S_{\ell}}$ acts on $\operatorname{span}\left\{\mathbf{1}_{m}\right\}^{\perp}$. To satisfy (4.12), we first note that $\operatorname{span}\left\{\mathbf{1}_{S_{k}}\right\}^{\perp}=\left\{u=\sum_{k} e_{k} \otimes u_{k}: u_{k} \in \mathbb{R}^{m}, \mathbf{1}_{m}^{T} u_{k}=0, \forall k\right\}$, where $e_{k}=e_{k}^{(m)}$.

In other words, $\operatorname{span}\left\{\mathbf{1}_{S_{k}}\right\}^{\perp}$ is the set of vectors $u$ such that each subvector $u_{S_{k}}$ sums to zero. Now, take $u \in \operatorname{span}\left\{\mathbf{1}_{S_{k}}\right\}^{\perp}$. Then, $u=\sum_{k} u_{S_{k}}=$ $\sum_{k} e_{k} \otimes u_{k}$, for some $\left\{u_{k}\right\} \subset \operatorname{span}\left\{\mathbf{1}_{m}\right\}^{\perp}$, and we have

$$
u^{T} \Lambda u=\sum_{k, \ell} u_{S_{k}}^{T} \Lambda u_{S_{\ell}}=\sum_{k, \ell} u_{k}^{T} \Lambda_{S_{k} S_{\ell}} u_{\ell}=\sum_{k} u_{k}^{T} \Lambda_{S_{k}} u_{k}+\sum_{k \neq \ell} u_{k}^{T} \Lambda_{S_{k} S_{\ell}} u_{\ell}
$$

Recall that $\Delta:=A-\mathbb{E}[A]$ where $[\mathbb{E} A]_{S_{k}}=p \boldsymbol{E}_{m}$ and $[\mathbb{E} A]_{S_{k} S_{\ell}}=q \boldsymbol{E}_{m}, k \neq \ell$. Then, $A_{S_{k}}=p \boldsymbol{E}_{m}+\Delta_{S_{k}}$ and from (10.1), we have $\Lambda_{S_{k}}=\mu \boldsymbol{E}_{m}+\operatorname{diag}^{*}\left(\nu_{S_{k}}\right)-$ $A_{S_{k}}$. It follows that

$$
u_{k}^{T} \Lambda_{S_{k}} u_{k}=u_{k}^{T}\left[(\mu-p) \boldsymbol{E}_{m}+\operatorname{diag}^{*}\left(\nu_{S_{k}}\right)-\Delta_{S_{k}}\right] u_{k}=u_{k}^{T}\left(\operatorname{diag}^{*}\left(\nu_{S_{k}}\right)-\Delta_{S_{k}}\right) u_{k}
$$


using the fact that $u_{k}^{T} \boldsymbol{E}_{m} u_{k}=\left(\mathbf{1}_{m}^{T} u_{k}\right)^{2}=0$. We also note from (10.2) that

$$
\operatorname{diag}^{*}\left(\nu_{S_{k}}\right)=\operatorname{diag}^{*}\left(\left[d\left(S_{k}\right)\right]_{S_{k}}\right)-\mu m I_{m} .
$$

On the other hand, for $k \neq \ell$, we have $\Lambda_{S_{k} S_{\ell}}=\mu \boldsymbol{E}_{m}-(A+\Gamma)_{S_{k} S_{\ell}}=$ $-B_{S_{k} S_{\ell}}$ from (10.1) and (11.4). To summarize,

$u^{T} \Lambda u=\sum_{k} u_{k}^{T}\left(\operatorname{diag}^{*}\left(\left[d\left(S_{k}\right)\right]_{S_{k}}\right)-\Delta_{S_{k}}\right) u_{k}-\mu m \sum_{k}\left\|u_{k}\right\|^{2}-\sum_{k \neq \ell} u_{k}^{T} B_{S_{k} S_{\ell}} u_{\ell}$.

To satisfy (4.12), we want $u^{T} \Lambda u$ to be big, which is the case if both $\mu$ and $\left\{B_{S_{k} S_{\ell}}\right\}$ are small. We are free to choose them subject to dual feasibility constraint $\Gamma \geq 0$, which translates to $\mu \boldsymbol{E}_{m}+B_{S_{k} S_{\ell}} \geq A_{S_{k} S_{\ell}}$. Our construction of $\Gamma_{S_{k} S_{\ell}}$ in (10.3) corresponds to $B_{S_{k} S_{\ell}}:=P_{\mathbf{1}_{\frac{1}{m}}} A_{S_{k} S_{\ell}} P_{\mathbf{1}_{m}^{\perp}}$. See also Remark 11.1 for a discussion of the trade-off involved.

Proof of Part (A). To verify dual feasibility, we use $P_{\mathbf{1}_{m}^{\perp}} e_{j}=e_{j}-\frac{1}{m} \mathbf{1}_{m}$ to write

$$
\begin{aligned}
{\left[\Gamma_{S_{k} S_{\ell}}\right]_{i j}=e_{i}^{T} \Gamma_{S_{k} S_{\ell}} e_{j} } & =\mu+\left(e_{i}-\frac{1}{m} \mathbf{1}_{m}\right)^{T} A_{S_{k} S_{\ell}}\left(e_{j}-\frac{1}{m} \mathbf{1}_{m}\right)-A_{i j} \\
& =\mu-\frac{1}{m} e_{i}^{T} A_{S_{k} S_{\ell}} \mathbf{1}_{m}-\frac{1}{m} \mathbf{1}_{m}^{T} A_{S_{k} S_{\ell}} e_{j}+\frac{1}{m^{2}} \mathbf{1}_{m}^{T} A_{S_{k} S_{\ell}} \mathbf{1}_{m} \\
& =\mu-\frac{1}{m}\left[d_{i}\left(S_{\ell}\right)+d_{j}\left(S_{k}\right)-d_{\mathrm{av}}\left(S_{k}, S_{\ell}\right)\right] \geq 0
\end{aligned}
$$

(A3) holds if $\left[\Gamma_{S_{k} S_{\ell}}\right]_{i j}>0$ for at least one $(i, j) \in S_{k} \times S_{\ell}$, for each pair $k \neq \ell$, which is equivalent to the stated condition.

Proof OF PART (B). To verify (4.12), we recall representation (11.6). By assumption $\operatorname{diag}^{*}\left(\left[d\left(S_{k}\right)\right]_{S_{k}}\right) \succeq \rho m I_{m}$ for all $k$. Also, using $B_{S_{k} S_{\ell}}=$ $P_{\mathbf{1}_{m}^{\perp}} A_{S_{k} S_{\ell}} P_{\mathbf{1}_{m}^{\perp}}$ we have

$u_{k}^{T} B_{S_{k} S_{\ell}} u_{\ell}=u_{k}^{T} P_{\mathbf{1}_{m}^{\perp}}\left(q \boldsymbol{E}_{m}+\Delta_{S_{k} S_{\ell}}\right) P_{\mathbf{1}_{\frac{1}{m}}} u_{\ell}=u_{k}^{T} P_{\mathbf{1}_{m}^{\perp}} \Delta_{S_{k} S_{\ell}} P_{\mathbf{1}_{m}^{\perp}} u_{\ell}=u_{k}^{T} \Delta_{S_{k} S_{\ell}} u_{\ell}$

for $\left\{u_{k}\right\} \subset \operatorname{span}\left\{\mathbf{1}_{m}\right\}^{\perp}$. From (11.6) it follows that

$$
\begin{aligned}
u^{T} \Lambda u & \geq \rho m \sum_{k}\left\|u_{k}\right\|^{2}-\sum_{k} u_{k}^{T} \Delta_{S_{k}} u_{k}-\mu m \sum_{k}\left\|u_{k}\right\|^{2}-\sum_{k \neq \ell} u_{k}^{T} \Delta_{S_{k} S_{\ell}} u_{\ell} \\
& =(\rho-\mu) m\|u\|^{2}-u^{T} \Delta u \\
& \geq[(\rho-\mu) m-\|\Delta\|]\|u\|^{2} .
\end{aligned}
$$


REMARK 11.1. The trade-off in choosing $\mu$ and $B_{S_{k} S_{\ell}}$ can be abstracted away in the following subproblem:

$$
h(\mu):=\min \left\{\|\widetilde{B}\|: \mu \boldsymbol{E}_{m}+\widetilde{B} \geq \widetilde{A}, \operatorname{range}(\widetilde{B}) \subset \operatorname{span}\left\{\mathbf{1}_{m}\right\}^{\perp}\right\}
$$

where $\widetilde{A} \in\{0,1\}^{m \times m}$ is a non-symmetric adjacency matrix (say, of a directed Erdos-Renyi graph with connection probability $q$ ). If $\mu=1$, one can take $\widetilde{B}=0$, hence $h(1)=0$. As one decreases $\mu$ from 1 , the feasible set of the problem shrinks until the problem becomes infeasible for some $\mu_{0} \in(0,1)$, if $\widetilde{A} \neq 0$. We have chosen $\widetilde{B}=P_{\mathbf{1}_{m}^{\perp}} \widetilde{A} P_{\mathbf{1}_{\frac{1}{m}}}$, essentially the largest choice, to make $\mu$ as small as possible. This might not in general be optimal. It would be interesting to study $h(\mu)$ more carefully. For example, another choice is $\widetilde{B}=P_{V} \widetilde{A} P_{V}$ where $V$ is a proper subspace of $\operatorname{span}\left\{\mathbf{1}_{m}\right\}^{\perp}$ of low dimension. This increases $\mu$, but decreases $h(\mu)$, helping us to better control the contributions of off-diagonal blocks in (11.6).

12. Probabilistic conditions for $\mathbf{P} \mathbf{P}^{\text {bal }}$. We will show when the construction of $(\mu, \nu, \Gamma)$ in (10.2) and (10.3) works for the balanced planted partition model, completing the proof of Theorem 4.1. We start we a consequence of Proposition 4.1.

Corollary 12.1. Let $A=\left(A_{i j}\right) \in\{0,1\}^{n \times n}$ be drawn from $\operatorname{PP}^{b a l}(p, q)$ with $p \geq\left(C^{\prime} \log m\right) / m$ and $q \geq\left(C^{\prime} \log n\right) / n$. Then, w.p. at least $1-c\left(K m^{-r}+\right.$ $\left.n^{-r}\right)$,

$$
\|A-\mathbb{E} A\| \leq C(\sqrt{p m}+\sqrt{q n}) .
$$

Proof. Let $\Delta:=A-\mathbb{E} A$ and decompose it into its diagonal and offdiagonal blocks. In particular, let $\boldsymbol{S}_{0}:=\operatorname{supp}\left(X_{0}\right)=\bigcup_{k} S_{k} \times S_{k}$ and let $\boldsymbol{S}_{0}^{c}$ be its complement. Then,

$$
\|\Delta\| \leq\left\|\boldsymbol{E}_{\boldsymbol{S}_{0}} \circ \Delta\right\|+\left\|\boldsymbol{E}_{\boldsymbol{S}_{0}^{c}} \circ \Delta\right\|=\max _{k}\left\|\Delta_{S_{k}}\right\|+\left\|\boldsymbol{E}_{\boldsymbol{S}_{0}^{c}} \circ \Delta\right\|
$$

$\boldsymbol{E}_{\boldsymbol{S}_{0}^{c} \circ \Delta}$ is an $n \times n$ matrix whose entries have variance $\leq q$, hence $\left\|\boldsymbol{E}_{\boldsymbol{S}_{0}^{c} \circ \Delta \| \leq}\right\|$ $C \sqrt{q n}$ w.p. at least $1-c n^{-r}$. Each $\Delta_{k}$ is an $m \times m$ matrix whose entries have variance bounded by $p$, hence $\left\|\Delta_{k}\right\| \leq C \sqrt{p m}$ w.p. at least $1-\mathrm{cm}^{-r}$, for each $k$. The result follows from union bound.

The following consequence of Bernstein's inequality summarizes the concentration of $d\left(S_{k}\right)$ around their mean. For simplicity, we will assume that the diagonal of $A$ is also filled with $\operatorname{Bern}(p)$ variates. This has no effect on 
the optimal primal solution due to the diagonal conditions $X_{i i}=1$. Recall that

$$
m K=n, \quad \bar{p}:=p m, \quad \bar{q}:=q m .
$$

Lemma 12.1. Let $\gamma:=\sqrt{\left(4 c_{1} \log n\right) / \bar{p}}$ and $\zeta:=\sqrt{\left(4 c_{2} \log n\right) / \bar{q}}$ and assume $\gamma, \zeta \in[0,3]$. Then,

$$
\begin{aligned}
& d_{i}\left(S_{k}\right) \geq \bar{p}(1-\gamma), i \in S_{k}, \forall k \quad \text { w.p. at least } 1-n^{-\left(c_{1}-1\right)} \text {, and } \\
& \left|d_{i}\left(S_{\ell}\right)-\bar{q}\right| \leq \zeta \bar{q}, \quad i \in S_{k}, \forall(k \neq \ell), \quad \text { w.p. at least } 1-2 m^{-1} n^{-\left(c_{2}-2\right)} \text {. }
\end{aligned}
$$

The proof is deferred to the Appendix 13. Assume now that the conditions of Lemma 12.1 (on $\gamma$ and $\zeta$ ) are met. Then w.h.p., $d_{\mathrm{av}}\left(S_{k}, S_{\ell}\right)$ is also in $[\bar{q}(1-\zeta), \bar{q}(1+\zeta)]$, for $k \neq \ell$, so that

$$
d_{i}\left(S_{\ell}\right)+d_{j}\left(S_{k}\right)-d_{\mathrm{av}}\left(S_{k}, S_{\ell}\right) \leq 2 \bar{q}(1+\zeta)-\bar{q}(1-\zeta)=\bar{q}(1+3 \zeta) .
$$

Thus, to satisfy (10.5), it is enough to have $\mu m \geq \bar{q}(1+3 \zeta)$. On the other hand, Lemma 12.1 implies that $m \rho:=\min _{k} \min _{i \in S_{k}} d_{i}\left(S_{k}\right) \geq \bar{p}-\bar{p} \gamma$. Then,

$$
\begin{aligned}
(\rho-\mu) m & \geq \bar{p}-\bar{p} \gamma-\bar{q}-3 \bar{q} \gamma \\
& \geq \bar{p}-\bar{q}-\sqrt{4 c_{1} \bar{p} \log n}-3 \sqrt{4 c_{2} \bar{q} \log n}
\end{aligned}
$$

By Corollary 12.1, w.h.p. $\|\Delta\| \leq C(\sqrt{\bar{p}}+\sqrt{\bar{q} K})$, where we have used $q n=$ $\bar{q} K$. Then, to satisfy (10.6), it is enough to have

$$
\bar{p}-\bar{q}-\sqrt{4 c_{1} \bar{p} \log n}-3 \sqrt{4 c_{2} \bar{q} \log n}>C(\sqrt{\bar{p}}+\sqrt{\bar{q} K})
$$

which is implied by

$$
\bar{p}-\bar{q}>\left(C+\sqrt{4 c_{1}}\right) \sqrt{\bar{p} \log n}+\left(C+3 \sqrt{4 c_{2}}\right) \sqrt{\bar{q} K \log n}
$$

in turn implied by

$$
\bar{p}-\bar{q}>C_{2}(\sqrt{\bar{p} \log n}+\sqrt{\bar{q} K \log n}) .
$$

Auxiliary conditions we needed on $\bar{p}$ and $\bar{q}$ were $\bar{p} \geq\left(4 c_{1} / 9\right) \log n$ and $\bar{q} \geq\left(4 c_{2} / 9\right) \log n$ from Lemma 12.1 and $\bar{p} \geq C^{\prime} \log m$ and $n q>C^{\prime} \log n$ from Corollary 12.1. We can drop the lower bounds on $q$ due to Corollary 4.2. The lower bounds on $\bar{p}$ are implied by $\bar{p} \geq\left(C^{\prime} \vee\left(4 c_{1} / 9\right)\right) \log n$. This completes the proof. To get to the form in which the theorem is stated, replace $c_{1}$ with $c_{1}+1$ and $c_{2}$ with $c_{2}+2$, and divide (12.1) by $\log n$. 
13. Proof of Lemma 12.1. We recall the following version of Bernstein inequality.

Proposition 13.1 (Bernstein). Let $\left\{X_{i}\right\}$ be independent zero-mean RVs, with $\left|X_{i}\right| \leq 1$ almost surely, and let $v:=\sum_{i} \mathbb{E}\left[X_{i}^{2}\right]$, then

$$
\mathbb{P}\left(\sum_{i=1}^{n} X_{i}>v t\right) \leq \exp [-v \phi(t)], t>0, \quad \text { where } \phi(t):=\frac{t^{2}}{2(1+t / 3)}
$$

For the first assertion, note that for $i \in S_{k}, d_{i}\left(S_{k}\right)=\sum_{j \in S_{k}} A_{i j}$ is a binomial random variable with mean $m p$ and variance $m p(1-p) \leq m p$. then applying Bernstein's with $v=m p$ and $t=\gamma$, we have $\mathbb{P}\left[d_{i}\left(S_{k}\right)-m p<\right.$ $-m p \gamma] \leq \exp (-m p \phi(\gamma))$. It follows from union bound that

$$
\mathbb{P}\left(\min _{k} \min _{i \in S_{k}} d_{i}\left(S_{k}\right) \geq \bar{p}(1-\gamma)\right) \geq 1-m K \exp (-\bar{p} \phi(\gamma))
$$

For $\gamma \in[0,3]$, we have $\phi(\gamma) \geq \gamma^{2} / 4$. It follows that $m K \exp (-\bar{p} \phi(\gamma)) \leq$ $n \exp \left(-\bar{p} \gamma^{2} / 4\right) \leq n n^{-c_{1}}$, proving the first assersion. The second assersion follows similarly, by noting that $d_{i}\left(S_{\ell}\right)$ is binomial with mean $\bar{q}=q m$ for $i \in S_{k}, k \neq \ell$. It follows from two-sided Bernstein and union bound that

$$
\begin{aligned}
\mathbb{P}\left(\max _{k \neq \ell} \max _{i \in S_{k}}\left|d_{i}\left(S_{\ell}\right)-\bar{q}\right| \leq \zeta \bar{q}\right) & \geq 1-\left[2\left(\begin{array}{c}
K \\
2
\end{array}\right) m\right] 2 \exp (-\bar{q} \phi(\zeta)) \\
& \geq 1-(m K)^{2} m^{-1} 2 \exp \left(-\bar{q} \zeta^{2} / 4\right) .
\end{aligned}
$$

The rest of the argument follows as before.

14. Proof of Proposition 5.1. The implication $(a) \Longrightarrow(b)$ follows since in the balanced case $\xi_{k}=1, \forall k$. Let us explain how part (b) implies part (c): Let $M:=\mathbb{E}[A]$ for a weakly but not strongly assortative block model. Let $\widetilde{M}$ be the matrix obtained from $M$ by setting all the diagonal blocks identically equal to one, except for one of the blocks that violates strong assortativity. Part (b) applies to $\widetilde{M}$ with $\left|I^{c}\right|=1$ and hence $\operatorname{SDP}_{\text {sol }}(\widetilde{M}) \neq\left\{X_{0}\right\}$. It then follows from Lemma 4.1 that $\operatorname{SDP}_{\text {sol }}(M) \neq$ $\left\{X_{0}\right\}$ which is the desired result.

The remainder of this section is devoted to proving part (a). We take dual variable $\Lambda$ be of the following form

$$
\Lambda=\sum_{k \in I} \lambda_{k}\left(-\boldsymbol{E}_{S_{k}}+n_{k} I_{S_{k}}\right), \quad \text { with } \lambda_{k} \geq 0 .
$$


In order to satisfy $\Lambda X=0$, it is enough to have $\Lambda \mathbf{1}_{S_{r}}=0, r \in I$. This holds for the form given in (14.1), namely, $\Lambda \mathbf{1}_{S_{r}}=\lambda_{r}\left(-\boldsymbol{E}_{S_{r}}+n_{r} I_{r}\right) \mathbf{1}_{S_{r}}=$ $\lambda_{r}\left(-n_{r} I_{S_{r}}+n_{r} I_{S_{r}}\right)=0$. We also assume the following form for $\Gamma$,

$$
\Gamma=\sum_{k \neq \ell} \rho_{k \ell} \boldsymbol{E}_{S_{k} S_{\ell}}+\sum_{k \notin I}\left[\gamma_{k} \boldsymbol{E}_{S_{k}}-\gamma_{k} I_{S_{k}}\right]
$$

For $k \in I$, we have $\alpha_{k k} \neq 0$, and (CSa) implies $\Gamma_{S_{k}}=0$. We also have $X_{i i}=1, \forall i$, hence $\Gamma_{i i}=0, \forall i$. The form given in (14.2) respects these conditions.

Let $M:=\mathbb{E}[A]$ and recall that $\Lambda=\mu \boldsymbol{E}_{n}+\operatorname{diag}^{*}(\nu)-(M+\Gamma)$, hence $(14.1)$ is equivalent to

$$
\begin{aligned}
\mu \boldsymbol{E}_{n_{k} n_{\ell}}-(M+\Gamma)_{S_{k} S_{\ell}} & =0, \quad k \neq \ell \\
\mu \boldsymbol{E}_{n_{k}}+\operatorname{diag}^{*}\left(\nu_{S_{k}}\right)-M_{S_{k}} & =-\lambda_{k} \boldsymbol{E}_{n_{k}}+\lambda_{k} n_{k} I_{n_{k}}, \quad k \in I \\
\mu \boldsymbol{E}_{n_{k}}+\operatorname{diag}^{*}\left(\nu_{S_{k}}\right)-(M+\Gamma)_{S_{k}} & =0, \quad k \notin I .
\end{aligned}
$$

Recall that $M_{S_{k} S_{\ell}}=q_{k \ell} \boldsymbol{E}_{n_{k} n_{\ell}}$ for $k \neq \ell$, and $M_{S_{k}}=p_{k} \boldsymbol{E}_{n_{k}}$. Hence, (14.3) is equivalent to $\forall(k \neq \ell) \rho_{k \ell}=\mu-q_{k \ell}$. Let us now simplify condition (14.4). Looking at the diagonal, we have $\mu+\nu_{i}-p_{k}=\left(n_{k}-1\right) \lambda_{k}, i \in S_{k}, k \in I$. Looking at the off-diagonal, we have $\lambda_{k}=p_{k}-\mu$. Hence, $\nu_{i}=n_{k} \lambda_{k}$ for $i \in$ $S_{k}, k \in I$. Now consider (14.5). For $k \notin I$, the diagonal gives $\mu+\nu_{i}-p_{k}=0$ since $A_{i i}=p_{k}$ and $\Gamma_{i i}=0$ (because of $X_{i i}=1$.) Hence, $\nu_{i}=p_{k}-\mu$ for $i \in S_{k}, k \notin I$. The off-diagonal gives $\gamma_{k}=\mu-p_{k}$. The following table summarizes these relationships:

$$
\begin{array}{l|l|l}
k \in I & k \notin I & \forall(k \neq \ell) \\
\hline \lambda_{k}=p_{k}-\mu & \rho_{k \ell}=\mu-q_{k \ell} \\
\nu_{i}=n_{k} \lambda_{k}, i \in S_{k} & \begin{array}{l}
\nu_{i}=p_{k}-\mu, i \in S_{k} \\
\gamma_{k}=\mu-p_{k}
\end{array} &
\end{array}
$$

(CSa) implies $(\forall k \notin I) \beta_{k} \gamma_{k}=0$, and $(\forall k, \ell \in I, k \neq \ell), \rho_{k \ell} \alpha_{k \ell}=0$. Together with dual feasibility, namely $\lambda_{k} \geq 0, \gamma_{k} \geq 0$, and $\rho_{k \ell} \geq 0$, we obtain the following restrictions on $\mu$,

\begin{tabular}{c|c|c}
$k \in I$ & $k \notin I$ & $\forall(k \neq \ell)$ \\
\hline$\mu<p_{k}$ & $\mu \geq p_{k}$ & $\mu \geq q_{k \ell}$ \\
& $\beta_{k}\left(\mu-p_{k}\right)=0$ & $\alpha_{k \ell}\left(\mu-q_{k \ell}\right)=0, k, \ell \in I$
\end{tabular}

It is interesting to note that when $\left\{q_{k \ell}, k \neq \ell\right\}$ are distinct (which is not assumed here), at most one of $\left\{\alpha_{k \ell}, k<\ell, k, \ell \in I\right\}$ is nonzero. This is 
enforced by the condition in the last row and column of (14.6), since $\mu$ can be equal to at most one of $q_{k \ell}$.

Recall that $\left(k_{0}, \ell_{0}\right):=\operatorname{argmax}_{k \neq \ell} q_{k \ell}$ which is assumed to be unique, and $I:=\left\{k: p_{k} \geq \max _{k \neq \ell} q_{k \ell}\right\}$. By the assumption of weak assortativity, $\left\{k_{0}, \ell_{0}\right\} \subset I$. Let $\mu=q_{k_{0} \ell_{0}}$. Then, the condition in the last row and column of (14.6) implies that $\alpha_{k \ell}=0, \forall k, \ell \in I \backslash\left\{k_{0}, \ell_{0}\right\}, k \neq \ell$, that is, only $\alpha_{k_{0} \ell_{0}}=\alpha_{\ell_{0} k_{0}}$ could be nonzero. Also, note that with this choice of $\mu$, the first row of (14.6) is satisfied.

Since $\mu>p_{k}$ for $k \in I^{c}:=[K] \backslash I=\left\{k: p_{k}<q_{k_{0} \ell_{0}}\right\}$, the condition in the second row and column of (14.6), implies that $\beta_{k}=0$ for $k \notin I$. It remains to verify primal feasibility, namely, $\left\langle X, \boldsymbol{E}_{n}\right\rangle=m n$. Recall that $n_{k}=\xi_{k} m$ with $\xi_{k} \geq 1\left(\right.$ since $\left.m=\min _{k} n_{k}\right)$. We need to have

$$
\left\langle X, \boldsymbol{E}_{n}\right\rangle=\sum_{k \in I} n_{k}^{2}+2 \alpha_{k_{0} \ell_{0}} n_{k_{0}} n_{\ell_{0}}+\sum_{k \notin I} n_{k}=n m
$$

Writing $n=\sum_{k} n_{k}$ and dividing by $m$, this is equivalent to

$$
\sum_{k \in I} \xi_{k}^{2}+2 \alpha_{k_{0} \ell_{0}} \xi_{k_{0}} \xi_{\ell_{0}}+\frac{1}{m} \sum_{k \notin I} \xi_{k}=\sum_{k} \xi_{k}
$$

Some algebra gives the expression $\alpha_{k_{0} \ell_{0}}=\alpha_{k_{0} \ell_{0}}^{*}$ where the latter is given in (5.2). Under the stated assumption, $\alpha_{k_{0} \ell_{0}} \in[0,1]$, the constructed solution is primal feasible and the proof is complete.

15. Proof of Proposition 6.1. We start by proving part (b). Part (a) then follows by simple modifications to the argument. Throughout, we mainly have the case $m=\min _{k} n_{k}$ in mind, which adds to the complexity in the construction of the primal-dual witness. When $m<\min _{k} n_{k}$, the set $I^{c}$ that appears below will be empty and the argument simplifies.

Let $\mathcal{L}_{2}(X)=X \mathbf{1}_{n}$ and $b_{2}=m \mathbf{1}_{n}$. The dual to problem (6.1) is

$$
\begin{array}{ll}
\max _{\Gamma, \rho, \nu, \mu} & -\left\langle\rho, b_{2}\right\rangle+\left\langle\nu, \mathbf{1}_{n}\right\rangle \\
\text { s.t. } & \Lambda:=\operatorname{diag}^{*}(\nu)-\mathcal{L}_{2}^{*}(\rho)-\left(A-\mu \boldsymbol{E}_{n}+\Gamma\right) \succeq 0 \\
& \Gamma \geq 0, \rho \geq 0
\end{array}
$$

Besides primal and dual feasibility we have the following complementary slackness conditions

$$
\begin{array}{c|c|c}
(\mathrm{CSa}) & (\mathrm{CSb}) & (\mathrm{CSc}) \\
\hline \Gamma_{i j} X_{i j}=0, \forall i, j & \Lambda X=0 & \rho_{i}\left[\mathcal{L}_{2}(X)-b_{2}\right]_{i}=0, \forall i
\end{array}
$$


Consider the potential primal solution given in (6.2) and note that it always satisfies $X_{i i}=1, X \succeq 0$ and $X \geq 0$. We can use (CSc) to make a reasonable choice of $\left\{\alpha_{k}\right\}$. For $i \in S_{k},\left[\mathcal{L}_{2}(X)\right]_{i}=\left(X \mathbf{1}_{n}\right)_{i}=\alpha_{k} n_{k}+\left(1-\alpha_{k}\right)$, hence $\rho_{i}\left[\mathcal{L}_{2}(X)-b_{2}\right]_{i}=0$, together with the corresponding dual feasibility, translate to

$$
\begin{array}{r}
\phi_{k}\left[\left(\alpha_{k} n_{k}+1-\alpha_{k}\right)-m\right]=0, \\
\alpha_{k} n_{k}+1-\alpha_{k} \geq m
\end{array}
$$

Note that if $n_{k}>m$, then setting $\alpha_{k}=1$ forces $\phi_{k}=0$, hence we lose the flexibility associated with $\phi_{k}$. This suggests that we should avoid setting $\alpha_{k}=1$, as much as possible, unless $n_{k}=m$. For simplicity, let $I_{1}:=I_{1}\left(k_{0}\right)$, and recall that $I:=\left\{k: n_{k}>m\right\}$ and $I_{1} \subset I$. Let $I_{2}:=I \backslash I_{1}$ and note that $\alpha_{k}$ given in part (b) of the proposition can be written as

$$
\alpha_{k}:= \begin{cases}\frac{m-1}{n_{k}-1}<1, & k \in I_{2} \\ 1, & k \in I^{c} \cup I_{1}\end{cases}
$$

where $I^{c}:=[n] \backslash I=\left\{k: n_{k}=m\right\}$. Note that this choice frees $\phi_{k}, \forall k$ to be any nonnegative number, except for $k \in I_{1}$ where we need $\phi_{k}=0$.

We now turn to the dual variables. Let us take $\Lambda$ to be of the form

$$
\Lambda=\sum_{k=1}^{K} \lambda_{k}\left(-\boldsymbol{E}_{S_{k}}+n_{k} I_{S_{k}}\right), \quad \lambda_{k} \geq 0 .
$$

$\Lambda$ is block diagonal, and the $k$ th block has eigenvalues $\lambda_{k}\left(0, n_{k}, n_{k}, \ldots, n_{k}\right)$. We will choose $\rho_{S_{k}}=\frac{1}{2} \phi_{k} 1_{S_{k}}$. Note that $\mathcal{L}_{2}^{*}(\rho)=\rho \mathbf{1}_{n}^{T}+\mathbf{1}_{n} \rho^{T}$, hence $\left[\mathcal{L}_{2}^{*}(\rho)\right]_{S_{k} S_{\ell}}=\frac{1}{2}\left(\phi_{k}+\phi_{\ell}\right) \boldsymbol{E}_{n_{l}, n_{\ell}}$ for all $k, \ell$. With $M:=\mathbb{E}[A]$, the following has to hold

$$
\begin{aligned}
\mu \boldsymbol{E}_{n_{k}, n_{\ell}}-\frac{1}{2}\left(\phi_{k}+\phi_{\ell}\right) \boldsymbol{E}_{n_{k}, n_{\ell}}-(M+\Gamma)_{S_{k} S_{\ell}} & =0, \quad k \neq \ell \\
\mu \boldsymbol{E}_{n_{k}}+\operatorname{diag}^{*}\left(\nu_{S_{k}}\right)-\phi_{k} \boldsymbol{E}_{n_{k}}-M_{S_{k}} & =\lambda_{k}\left(-\boldsymbol{E}_{n_{k}}+n_{k} I_{n_{k}}\right) .
\end{aligned}
$$

In deriving (15.3), we have used $\Gamma_{S_{k}}=0, \forall k$ which follows from the particular choice of $X$ in (6.2) and (CSa). Let $\psi_{k}:=\mu-\phi_{k}$. Using $M_{S_{k} S_{\ell}}=$ $q_{k \ell} \boldsymbol{E}_{n_{k} n_{\ell}}, k \neq \ell$ and $M_{S_{k}}=p_{k} \boldsymbol{E}_{n_{k}}$, we arrive at

$$
\begin{aligned}
\Gamma_{S_{k} S_{\ell}} & =\left[\frac{1}{2}\left(\psi_{k}+\psi_{\ell}\right)-q_{k \ell}\right] \boldsymbol{E}_{n_{k}, n_{\ell}}, \\
\psi_{k}+\nu_{i}-p_{k} & =\left(n_{k}-1\right) \lambda_{k}, \quad i \in S_{k} \\
\psi_{k}-p_{k} & =-\lambda_{k}
\end{aligned}
$$


where the last two equalities are obtained by considering the diagonal and off-diagonal elements in (15.3). It follows that $\lambda_{k}=p_{k}-\psi_{k}$ and $\nu_{i}=$ $n_{k} \lambda_{k}, i \in S_{k}$.

Dual feasibility implies

$$
\begin{aligned}
\phi_{k}=\mu-\psi_{k} & \geq 0, \forall k \\
\frac{1}{2}\left(\psi_{k}+\psi_{\ell}\right)-q_{k \ell} & \geq 0, \quad \forall k \neq \ell \\
\lambda_{k}=p_{k}-\psi_{k} & \geq 0, \quad \forall k
\end{aligned}
$$

(CSb), namely, $\Lambda X=0$, translates to $\lambda_{k}\left(1-\alpha_{k}\right)=0$, since $\boldsymbol{E}_{S_{k}}\left(-\boldsymbol{E}_{S_{k}}+\right.$ $\left.m_{k} I_{S_{k}}\right)=0$ implies $\Lambda X=\sum_{k} \lambda_{k}\left(1-\alpha_{k}\right)\left(-\boldsymbol{E}_{S_{k}}+n_{k} I_{S_{k}}\right)$. In particular, for $k \in I_{2}$, we have $\alpha_{k}<1$, hence $\lambda_{k}=0$; otherwise $\lambda_{k}$ is free to be any nonnegative number. To summarize, $(\mathrm{CSb})$ and (CSc) impose the following restrictions on the dual variables

$$
\left(\forall k \in I_{1}\right) \phi_{k}=0, \quad\left(\forall k \in I_{2}\right) \lambda_{k}=0 .
$$

Recall the inequality $q_{k \ell} \leq \frac{1}{2}\left(q_{k}^{*}+q_{\ell}^{*}\right), \forall k \neq \ell$. It follows that by choosing $\psi_{k} \geq q_{k}^{*}, \forall k$, we can satisfy (15.5). To satisfy (15.4), (15.6) and (15.7), we need

$$
\left(\forall k \in I_{1}\right) \psi_{k}=\mu \leq p_{k}, \quad\left(\forall k \in I_{2}\right) \psi_{k}=p_{k} \leq \mu, \quad\left(\forall k \in I^{c}\right) \psi_{k} \leq \min \left\{p_{k}, \mu\right\}
$$

where we note that $I_{1}, I_{2}, I^{c}$ form a partition of $[K]$. Thus, it is enough to have

$$
\left(\forall k \in I_{1}\right) \mu \in\left[q_{k}^{*}, p_{k}\right], \quad\left(\forall k \in I_{2}\right) \mu \geq p_{k}, \quad\left(\forall k \in I^{c}\right) q_{k}^{*} \leq \min \left\{p_{k}, \mu\right\}
$$

Since $\mu \in J_{k_{0}} \subset \bigcap_{k \in I_{1}\left(k_{0}\right)}\left[q_{k}^{*}, p_{k}\right]$, we have $\mu \in\left[q_{k}^{*}, p_{k}\right]$ for all $k \in I_{1}=I_{1}\left(k_{0}\right)$. Since we have taken $\mu \geq p_{k_{0}+1}$, we have $\mu \geq p_{k}$ for all $k \geq k_{0}+1$ due to th assumed ordering of $\left\{p_{k}\right\}$. In particular, $\mu \geq p_{k}$ for all $k \in I_{2}$. For $k \in I^{c}$, either $k \leq k_{0}$, in which case $\mu \in\left[q_{k}^{*}, p_{k}\right]$, i.e. $q_{k}^{*} \leq \mu=\min \left\{p_{k}, \mu\right\}$, or we have $k \geq k_{0}+1$ in which case $\mu \geq p_{k}$, hence $q_{k}^{*} \leq p_{k}=\min \left\{\mu, p_{k}\right\}$. Thus, all the conditions in (15.9) are met and the proof is complete.

Proof of part (a). The argument here is similar to that of part (b). In addition to setting $\mu=0$, the main difference is that (CSc) and dual feasibility condition $\rho_{i} \geq 0, \forall i$ is replaced by the single primal feasibility condition $\mathcal{L}_{2}(X)-b_{2}=0$. Note that there is no nonnegativity assumption on $\rho$ anymore. The argument goes true if we take $I_{1}=\emptyset$ and $I_{2}=I$, which ensures 
that $X \mathbf{1}_{n}-m \mathbf{1}_{n}=0$. We now have $\psi_{k}=-\phi_{k}$ and the dual feasibility conditions reduce to (15.5) and (15.6). Furthurmore, (15.7) is simplified to $(\forall k \in I) \lambda_{k}=0$, since only (CSb) is present. Thus, it is enough to have $\psi_{k} \geq q_{k}^{*}$ for all $k$ and

$$
(\forall k \in I) \psi_{k}=p_{k}, \quad\left(\forall k \in I^{c}\right) \psi_{k} \leq p_{k} .
$$

Since $q_{k}^{*} \leq p_{k}, \forall k$, by assumption, it is clearly possible to choose $\psi_{k}$ to satisfy these conditions.

16. Implementation of SDP-1. It is straightforward to adapt a first order method to solve the SDP-1 problem (3.5). We briefly discuss the implementation of an ADMM solver [16]. We start by rewriting the problem as

$$
\inf _{X}\left\{-\langle A, X\rangle+\delta_{\{\widetilde{\mathcal{L}}(X)=\widetilde{b}\}}+\delta_{\{Z \geq 0\}}+\delta_{\{Y \succeq 0\}}\right\} \quad \text { s.t. } X=Z, X=Y,
$$

where $\delta_{S}$ is the indicator of set $S$ defined by $\delta_{S}(x)=0$ if $x \in S$ and $=\infty$ otherwise, and $\widetilde{\mathcal{L}}: \mathbb{R}^{n \times n} \rightarrow \mathbb{R}^{2 n}$ is a linear operator such that $\widetilde{\mathcal{L}}(X)=\widetilde{b}$ collects the affine constraints in (3.5). More precisely, for $i=1, \ldots, n$, we take $[\widetilde{\mathcal{L}}(X)]_{i}=\left\langle X, H_{i}\right\rangle$ and $[\widetilde{\mathcal{L}}(X)]_{i+n}=\left\langle X, F_{i}\right\rangle$. Here, $H_{i}$ is a symmetric matrix with 1 in the off-diagonal elements of the $i$-th column and row, and 0 everywhere else. $F_{i}$ is a matrix with element $(i, i)$ equal to 1 and 0 everywhere else. Finally, $\widetilde{b}_{i}=2((n / K)-1)$ for $i=1, \ldots, n$ and $b_{i}=1$ otherwise. $(\widetilde{\mathcal{L}}$ is a variation of $\mathcal{L}$ that appears in Section 4.2.2. It is chosen so that $H_{i}$ is orthogonal to $F_{j}$ for all $i, j$. However, $\{\widetilde{\mathcal{L}}(X)=\widetilde{b}\}$ and $\{\mathcal{L}(X)=b\}$ describe the same affine subspace.)

The only real work in deriving ADMM updates is to find the projection operator $\Pi_{\mathcal{A}}$ for $\mathcal{A}:=\{X: \widetilde{\mathcal{L}}(X)=\widetilde{b}\}$. For any $Y$, this projection is given by

$$
\Pi_{\mathcal{A}}(Y):=Y-\widetilde{\mathcal{L}}^{*}\left(\widetilde{\mathcal{L}} \widetilde{\mathcal{L}}^{*}\right)^{-1}[\widetilde{\mathcal{L}}(Y)-\widetilde{b}]
$$

Note that $\left\langle H_{i}, F_{j}\right\rangle=0$ for all $i, j=1, \ldots, n$. Hence, $\widetilde{\mathcal{L}} \widetilde{\mathcal{L}}^{*}$ is block diagonal with two blocks $\left(\left\langle H_{i}, H_{j}\right\rangle\right)=2\left[(n-2) I_{n}+\mathbf{1}_{n} \mathbf{1}_{n}^{T}\right]$ and $\left(\left\langle F_{i}, F_{j}\right\rangle\right)=I_{n}$. It follows that

$$
\left(\widetilde{\mathcal{L}} \widetilde{\mathcal{L}}^{*}\right)^{-1}=\operatorname{diag}\left(\frac{1}{2(n-2)}\left[I_{n}-\frac{\mathbf{1}_{n} \mathbf{1}_{n}^{T}}{2 n-2}\right], I_{n}\right)
$$

We also have $\widetilde{\mathcal{L}}^{*}(\widetilde{\mu}, \nu)=\sum_{i} \widetilde{\mu}_{i} E_{i}+\sum_{i} \nu_{i} F_{i}=\left(\widetilde{\mu}_{i}+\widetilde{\mu}_{j}\right)_{i \neq j}+\operatorname{diag}(\nu)$, which gives a complete recipe to compute $\Pi_{\mathcal{A}}(Y)$. Note that due to the simplicity of $\left(\widetilde{\mathcal{L}} \widetilde{\mathcal{L}}^{*}\right)^{-1}$ and $\widetilde{\mathcal{L}}^{*}$, implementing this projection has essentially the same computational cost as projecting onto an affine set with two constraints 
$\left\{X: \operatorname{tr}(X)=n,\left\langle\boldsymbol{E}_{n}, X\right\rangle=n^{2} / K\right\}$, which is needed for implementing SDP-2.

The ADMM updates are easily derived to be

$$
\begin{aligned}
X^{k+1} & =\Pi_{\mathcal{A}}\left(\frac{1}{2}\left(Z^{k}-U^{k}+Y^{k}-V^{k}+\frac{1}{\rho} A\right)\right), \\
Z^{k+1} & =\max \left\{0, X^{k+1}+U^{k}\right\}, \quad Y^{k+1}=\Pi_{\mathbb{S}_{+}^{n}}\left(X^{k+1}+V^{k}\right), \\
U^{k+1} & =U^{k}+X^{k+1}-Z^{k+1}, \quad V^{k+1}=V^{k}+X^{k+1}-Y^{k+1} .
\end{aligned}
$$

where $\Pi_{\mathbb{S}_{+}^{n}}$ is the projection onto the PSD cone $\mathcal{S}_{+}^{n}$, which can be done by truncating to nonnegative eigenvalues. The ADMM updates for SDP-2 and SDP-3 can be derived similarly, as in [18].

17. Details on Figure 1: comparing the theoretical predictions with empirical results. Figure 1 provides an illustration of the contents of Propositions 5.1 and 6.1. The results are obtained by numerically solving the SDPs. Here, we explain how they match with our theoretical results. The leftmost panel corresponds to the mean matrix $M=\mathbb{E}[A]$ of a weakly assortative block model, randomly picked among such models. The specific edge probability matrix is as follows:

$\begin{array}{llllll}0.670 & 0.072 & 0.020 & 0.023 & 0.186 & 0.187 \\ 0.072 & 0.570 & 0.521 & 0.016 & 0.360 & 0.107 \\ 0.020 & 0.521 & 0.555 & 0.048 & 0.311 & 0.188 \\ 0.023 & 0.016 & 0.048 & 0.494 & 0.081 & 0.137 \\ 0.186 & 0.360 & 0.311 & 0.081 & 0.475 & 0.031 \\ 0.187 & 0.107 & 0.188 & 0.137 & 0.031 & 0.195\end{array}$.

There are six blocks of sizes $\underline{\boldsymbol{n}}=(10,10,5,20,10,10)$. The parameters $p_{k}$ and $q_{k}^{*}=\max _{\ell \neq k} q_{\ell: k \ell}$, for each of the $K=6$ blocks are as follows

$$
\begin{array}{lllllll}
q_{k}^{*} & 0.187 & 0.521 & 0.521 & 0.137 & 0.360 & 0.188 \\
p_{k} & 0.670 & 0.570 & 0.555 & 0.494 & 0.475 & 0.195
\end{array} .
$$

where the overall maximum of the off-diagonal entries is $\max _{k} q_{k}^{*}=0.521$. It is clear that the last three blocks violate strong associativity.

We can use part (a) of Proposition 5.1 to predict the behavior of SDP$2^{\prime}$. Note that for this example, $\left(k_{0}, \ell_{0}\right):=\operatorname{argmax}_{k<\ell} q_{k \ell}=(2,3)$. We have $m=\min _{k} n_{k}=5$, hence $\left(\xi_{k}\right)=(2,2,1,4,2,2)$ where $\xi_{k}=n_{k} / m, I=\{k$ : $\left.p_{k} \geq q_{k_{0} \ell_{0}}=0.521\right\}=\{1,2,3\}, \xi_{k_{0}}=2$ and $\xi_{\ell_{0}}=1$. It then follows that

$$
\alpha_{k_{0} \ell_{0}}^{*}=\frac{1}{2(2 \cdot 1)}\left[\left(1-\frac{1}{5}\right)(4+2+2)-(2 \cdot 1+2 \cdot 1+1 \cdot 0)\right]=0.6 .
$$


Since $\alpha_{k_{0} \ell_{0}}^{*} \in[0,1]$, the conditions of part (a) of Proposition 5.1 are met and the solution is of the form (5.2) with $\alpha_{1}=\alpha_{2}=\alpha_{3}=1, \alpha_{23}=\alpha_{32}=0.6$ and $\beta_{k}=0$ for $k=4,5,6$.

Let us now apply Proposition 6.1 to predict the behavior of SDP-13. Recall that $J_{k}=\bigcap_{r=1}^{k}\left[q_{r}^{*}, p_{r}\right]$ which in this case gives

\begin{tabular}{c|cccccc}
$k$ & 1 & 2 & 3 & 4 & 5 & 6 \\
\hline \multirow{2}{*}{$J_{k}$} & 0.187 & 0.521 & 0.521 & $\emptyset$ & $\emptyset$ & $\emptyset$
\end{tabular}.

We have $k_{0}=\max \left\{k: J_{k} \neq \emptyset\right\}=3$ and we can apply SDP-13 with any $\mu \in J_{3} \cap\left[p_{4}, 1\right]=[0.521,0.555] \cap[0.494,1]=[0.521,0.555]$. The images in Figure 1 are generated with $\mu=0.55$ and $m=\min _{k} n_{k}=5$. We have $I=$ $\left\{k: n_{k}>m\right\}=\left\{k: \xi_{k}>1\right\}=\{1,2,4,5,6\}$, hence $I_{1}\left(k_{0}\right)=I_{1}(3)=\{1,2\}$. Proposition 6.1(b) now applies and we have a solution of the form (6.2) with

\begin{tabular}{c|cccccc}
$k$ & 1 & 2 & 3 & 4 & 5 & 6 \\
\hline$\alpha_{k}$ & 1 & 1 & 1 & 0.211 & 0.444 & 0.444.
\end{tabular}

Note that we have three perfectly recovered blocks in the sense discussed after Proposition 6.1.

Finally, part (a) of Proposition 6.1 predicts that SDP-1, applied with $m=5$, has a solution of the form (6.2) with

\begin{tabular}{c|cccccc}
$k$ & 1 & 2 & 3 & 4 & 5 & 6 \\
\hline$\alpha_{k}$ & 0.444 & 0.444 & 1 & 0.211 & 0.444 & 0.444.
\end{tabular}

All the above predictions match what is empirically reported in Figure 1. We also note that although the behavior of SDP-3 is not mentioned in Propositions 5.1 and 6.1 , that solution can also be predicted by careful examination of the proofs.

18. Details on Figure 2: the bias of normalized mutual information for large $\boldsymbol{K}$. Top left panel in Figure 6 shows the average (empirical) NMI of random guessing as a function of $K$. Contrary to popular belief, the empirical NMI does not automatically adjust so that random guessing corresponds to zero NMI; unless $K$ is small. It is designed to do so based on population quantities, but for the population quantities to be accurately approximated by empirical ones, one needs concentration of the counts in the confusion matrix around their means, which does not happen unless $n / K$ is sufficiently large. Figure 6 shows the plots of Figure 2 after adjusting for random guessing by subtracting the corresponding average NMI. As one would expect, the dip in the curves goes away after the adjustment. 

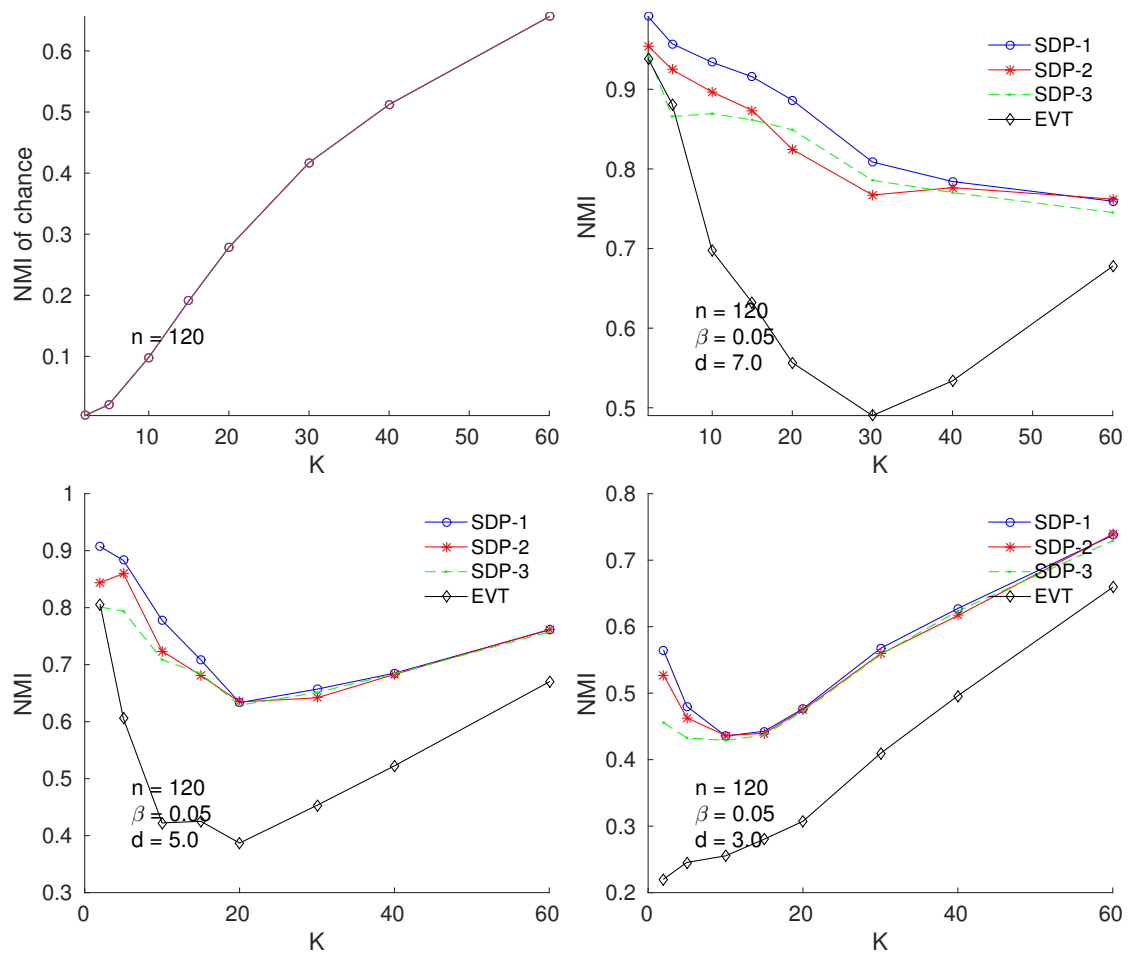

Fig 6: Top left: Average NMI of random guessing (or chance). The other three plots correspond to those of Figure 2 but with raw NMI (no adjustment for chance). 
Department of Statistics

UCLA

Los Angeles, CA 90095

E-MAIL: aaamini@ucla.edu
Department of Statistics UNIVERSITY OF MICHIGAN

Ann Arbor, MI 48109-1107

E-MAIL: elevina@umich.edu 